\title{
automatica
}

\section{Continuous pole placement for delay equations}

\author{
W. Michiels *, K. Engelborghs, P. Vansevenant, D. Roose \\ Department of Computer Science, Katholieke Universiteit Leuven, Celestijnenlaan 200A, B-3001 Leuven, Belgium
}

Received 6 October 2000; received in revised form 6 July 2001; accepted 15 October 2001

\begin{abstract}
In this paper, we describe a stabilization method for linear time-delay systems which extends the classical pole placement method for ordinary differential equations. Unlike methods based on finite spectrum assignment, our method does not render the closed loop system, finite dimensional but consists of controlling the rightmost eigenvalues. Because these are moved to the left half plane in a (quasi-)continuous way, we refer to our method as continuous pole placement. We explain the method by means of the stabilization of a linear finite dimensional system in the presence of an input delay and illustrate its applicability to more general stabilization problems. (C) 2002 Elsevier Science Ltd. All rights reserved.
\end{abstract}

Keywords: Delay equations; Feedback stabilization; Pole assignment

\section{Introduction}

The stabilization of linear time-delay systems has been studied extensively in the literature. Existing stabilization methods include those based on finite spectrum assignment (Brethé \& Loiseau, 1998; Manitius \& Olbrot, 1979; Olbrot, 1978; Wang, Lee, \& Tan, 1999), methods using an algebraic Riccati approach (where stability conditions are expressed by the solvability of a Riccati equation or by the feasibility of a linear matrix inequality, see Dion, Dugard, and Fliess (1998) and the references therein) and variants of the Smith predictor (see Palmor (1996), Chap. 10 for an overview).

In this paper we develop a numerical stabilization method which is related to the classical pole-placement method for ordinary differential equations (Ackermann, 1972). The approach, which starts from the state space description of a linear time-delay system, is mixed analytical/numerical and makes use of a recently developed method (Engelborghs \& Roose, 1999) for the determination of the rightmost eigenvalues of a linear time-delay system.

\footnotetext{
This paper was not presented at any IFAC meeting. This paper was recommended for publication in revised form by Associate Editor Irene Lasiecka under the direction of Editor Roberto Tempo.

* Corresponding author.

E-mail address: wim.michiels@cs.kuleuven.ac.be (W. Michiels).
}

As an illustration of our method, we study the stabilization of the system

$\dot{x}=A x+B u(t-\tau), \quad A \in \mathbb{R}^{n \times n}, B \in \mathbb{R}^{n \times 1}$,

where $x \in \mathbb{R}^{n}$ is the state, $u \in \mathbb{R}$ is the input and $\tau \geqslant 0$ represents an input delay. We use a linear static state feedback controller,

$u=K^{\mathrm{T}} x, \quad K \in \mathbb{R}^{n \times 1}$.

The control law (2) is not only motivated by the fact that it reveals the link between our stabilization method and the classical pole placement method, and allows to obtain some nice theoretical results. We will also show that for system (1), a static state feedback controller leads to stabilizability properties which are comparable to those of methods based on finite spectrum assignment, when small perturbations are taken into account. Furthermore, the extension to the output feedback case is straightforward.

The structure of the paper is as follows. First, we study theoretical stabilizability properties of system (1)-(2) and motivate the use of control law (2), thereby explaining the ideas behind the continuous pole placement method. Then we study the stabilization method in detail and apply it to a (numerical) example. After a discussion of the output-feedback case, we end with some numerical examples illustrating the applicability of our method to more general types of delay equations involving delays in both state and control variables, and multiple feedbacks. 


\section{Preliminaries}

The state of the delay equation

$\dot{x}=A x+A_{d} x(t-\tau), \quad x \in \mathbb{R}^{n}, A, A_{d} \in \mathbb{R}^{n \times n}$,

at time $t$ is given by a function segment $x_{t} \in$ $\mathscr{C}\left([-\tau, 0], \mathbb{R}^{n}\right)$ defined as

$x_{t}(\theta)=x(t+\theta), \quad \theta \in[-\tau, 0]$.

Here $\mathscr{C}\left([-\tau, 0], \mathbb{R}^{n}\right)$ is the Banach space of continuous functions mapping the delay-interval $[-\tau, 0]$ into $\mathbb{R}^{n}$ and equipped with the supremum-norm $\|.\|_{s}$. A solution is uniquely defined by specifying as initial condition a function segment $x_{0} \in \mathscr{C}\left([-\tau, 0], \mathbb{R}^{n}\right)$. Note that the delay equation can be considered as an evolution equation over this space. Therefore, delay equations belong to the very broad class of functional differential equations (Hale \& Verduyn Lunel, 1993; Kolmanovskii \& Myshkis, 1999; Kolmanovskii \& Nosov, 1986).

Stability definitions for (3) are analogous to the ODE case (Dugard \& Verriest, 1998; Kolmanovskii \& Nosov, 1986). The zero solution of (3) is asymptotically stable when

$\forall \varepsilon>0, \quad \exists \delta>0:\left\|x_{0}\right\|_{s} \leqslant \delta \rightarrow\left\|x_{t}\right\|_{s} \leqslant \varepsilon, \quad \forall t \geqslant 0$

and

$\lim _{t \rightarrow \infty} x(t)=0, \quad \forall x_{0} \in \mathscr{C}\left([-\tau, 0], \mathbb{R}^{n}\right)$.

This is equivalent with the fact that all eigenvalues, i.e. the roots of the characteristic equation,

$\operatorname{det}\left(\lambda I-A-A_{d} \mathrm{e}^{-\lambda \tau}\right)=0$,

are in the open left half plane. Condition (4) is equivalent to requiring the existence of a nonzero vector $v \in \mathbb{C}^{n \times 1}$ such that $\left(\lambda I-A-A_{d} \mathrm{e}^{-\lambda \tau}\right) v=0$. The function segment $v \mathrm{e}^{\lambda \theta}, \theta \in[-\tau, 0]$ is the eigenfunction corresponding to the eigenvalue $\lambda$. Eq. (4) is transcendental and has infinitely many solutions. However, the number of eigenvalues to the right of any vertical line, $\mathfrak{R}(\lambda) \geqslant r$, with $r \in \mathbb{R}$, is finite, and hence $-\infty$ is the only accumulation point for the real parts of the eigenvalues (Dugard \& Verriest, 1998; Hale \& Verduyn Lunel, 1993).

In the rest of paper we make the following assumption:

Assumption 2.1. The pair $(A, B)$ in (1) is controllable.

This allows to bring the closed loop system (1)-(2), after a similarity transformation $z=T x$, into the control canonical form:

$\dot{z}=A_{c} z+B_{c} K_{c}^{\mathrm{T}} z(t-\tau)$ or

$$
\begin{aligned}
\dot{z}= & {\left[\begin{array}{ccccc}
0 & 1 & & & \\
& 0 & 1 & & \\
& & \ddots & \ddots & \\
& & & 0 & 1 \\
-a_{1} & -a_{2} & \ldots & & -a_{n}
\end{array}\right] z(t) } \\
& +\left[\begin{array}{c}
0 \\
\vdots \\
0 \\
-1
\end{array}\right]
\end{aligned}
$$

Hence the eigenvalues of (1)-(2) satisfy,

$$
\begin{aligned}
H(\lambda) \triangleq & \lambda^{n}+\left(a_{n}+k_{n} \mathrm{e}^{-\lambda \tau}\right) \lambda^{n-1}+\cdots \\
& +\left(a_{2}+k_{2} \mathrm{e}^{-\lambda \tau}\right) \lambda+\left(a_{1}+k_{1} \mathrm{e}^{-\lambda \tau}\right)=0 .
\end{aligned}
$$

\section{Motivation}

The problem of the stabilization of system (1) with a feedback controller of form (2) is hard because its design involves the determination of only $n$ parameters, while the closed loop system has an infinite number of eigenvalues. On the other hand the proposed controller structure is very simple and easy to implement. Furthermore, recent research (Engelborghs, Dambrine, \& Roose, 2001; Van Assche, Dambrine, Lafay, \& Richard, 1999) shows that more sophisticated controllers, which are based on prediction and yield only a finite number of closed loop eigenvalues, may be sensitive to arbitrary small implementation errors and suffer from the same limitations as controller (2) from a practical point of view. This will be illustrated with a one-dimensional example in Section 3.3.

\subsection{A finite-dimensional controller for an infinite-dimensional problem}

The classical pole placement algorithm (Ackermann, 1972) cannot be applied directly to the time-delay case because the characteristic equation (7) has infinitely many solutions while the number of degrees of freedom in the controller is $n$. A direct placement of $n$ eigenvalues is always possible. This follows from the linearity of the characteristic equation (7) w.r.t. the components of $K_{c}$. Indeed Eq. (7) can be rewritten as

$$
\left[\begin{array}{lllll}
1 & \lambda & \lambda^{2} & \ldots & \lambda^{n-1}
\end{array}\right]\left[\begin{array}{llll}
k_{1} & k_{2} & \ldots & k_{n}
\end{array}\right]^{\mathrm{T}}=-\bar{p}(\lambda) \mathrm{e}^{\lambda \tau},
$$

where $\bar{p}(\lambda)=\sum_{i=1}^{n} a_{i} \lambda^{i-1}+\lambda^{n}$. When forcing $n$ different eigenvalues $\lambda_{1}, \ldots, \lambda_{n}$ to satisfy this equation, a linear 
system of equations with unknown $K_{c}$ and a regular Jacobian matrix (a Vandermonde matrix) is obtained. However, by placing $n$ eigenvalues, which determines the complete spectral picture, control is lost over the position of the other ones, and these may cause instability.

Our method is based on the fact that, unlike for neutral equations (Michiels, Engelborghs, Roose, \& Dochain, 1998), the number of unstable eigenvalues is always finite (Hale \& Verduyn Lunel, 1993, Lemma I.4.1), and on the availability of a software tool to calculate the rightmost eigenvalues (Engelborghs, 2000). Once we have detected unstable eigenvalues, our strategy consists of moving them to the left half plane by applying small changes to the controller gain $K$, and meanwhile monitoring the other eigenvalues with large real part. Because the eigenvalues move continuously w.r.t. changes in the feedback gain, see Theorem 8 in the appendix, we refer to our method as the continuous pole placement method. Before describing the method in more detail in the next section, we introduce some theorems concerning the limitations and difficulties of linear state feedback control in the presence of input delays.

When the uncontrolled system (1) has eigenvalues in the closed RHP, the destabilizing effect of a time delay is illustrated with the fact that no fixed feedback-gain $K$ is able to achieve stabilization for all values of the time delay $\tau$, a consequence of the following theorem from in 't Hout (1994). By $\sigma(\cdot)$ we denote the spectrum and by $r_{\sigma}(\cdot)$ the spectral radius.

Theorem 1. Delay-independent stability:

$$
\begin{aligned}
\dot{x}(t)= & A x(t)+A_{d} x(t-\tau) \text { asymptotically stable for all } \\
& \tau \geqslant 0 \\
& \Re(\sigma(A))<0 \text { and } \sup _{\omega \in \mathbb{R}} r_{\sigma}\left((j \omega I-A)^{-1} A_{d}\right) \leqslant 1 .
\end{aligned}
$$

We can strengthen this result: when $A$ has eigenvalues in the open right half plane, even semi-global stabilization in the delay is not possible.

Theorem 2. When A has eigenvalues in the open RHP, the system $\dot{x}=A x+B u(t-\tau)$ cannot be asymptotically stabilized semi-globally in the delay using state feedback $u=K x$, i.e.

$\exists \bar{\tau}<\infty$ such that

$\forall K \in \mathbb{R}^{n}, \exists \tau \leqslant \bar{\tau}: \dot{x}=A x+B K^{\mathrm{T}} x(t-\tau)$ is not

asymptotically stable.

Proof. Suppose that the theorem does not hold, i.e. there exist sequences $\left\{K_{n}\right\}_{n \geqslant 1}$ and $\left\{\tau_{n}\right\}_{n \geqslant 1}$ with $\lim _{n \rightarrow \infty} \tau_{n}=\infty$ such that the feedback $u=K_{n} x$ asymptotically stabilizes the system for $0 \leqslant \tau \leqslant \tau_{n}$. We show that this always leads to a contradiction. We make distinction between two cases.

Case 1: the sequence $\left\{\left\|K_{n}\right\|\right\}_{n \geqslant 1}$ is bounded. Because all elements of $\left\{K_{n}\right\}_{n \geqslant 1}$ belong to a compact region in $\mathbb{R}^{n}$, there exists a converging subsequence with limit $K$. Denote by $\lambda_{0}$ an eigenvalue of $A$ in the open right half plane and define the disc $D=\left\{\lambda:\left|\lambda-\lambda_{0}\right| \leqslant \Re\left(\lambda_{0}\right) / 2\right\}$. The sequence of analytic functions $\left\{f\left(\lambda, \tau_{n}\right)\right\}_{n \geqslant 1}=\{\operatorname{det}(\lambda I-$ $\left.\left.A-B K^{\mathrm{T}} \mathrm{e}^{-\lambda \tau_{n}}\right)\right\}_{n \geqslant 1}$ converges uniformly on the disc $D$ to the function $f(\lambda)=\operatorname{det}(\lambda I-A)$. We can apply Lemma 7 of the appendix, which states that for large $n$, the number of zeros of $f\left(\lambda, \tau_{n}\right)$ and $f(\lambda)$ in $D$ are equal. As a consequence, the system $\dot{x}=A x+B K^{\mathrm{T}} x\left(t-\tau_{n}\right)$ has an eigenvalue in the open RHP for large values of $n$. Hence, there exists an integer $\hat{n}$ such that $\dot{x}=A x+B K x\left(t-\tau_{\hat{n}}\right)$ has an eigenvalue in the open RHP, whereas $\dot{x}=A x+B K_{n} x(t-$ $\left.\tau_{\hat{n}}\right)$ is asymptotically stable for large $n$, since $\left\{K_{n}\right\}_{n \geqslant 1}$ asymptotically stabilizes the system for $\tau \in\left[0, \tau_{n}\right]$. Because $\left\{K_{n}\right\}_{n \geqslant 1}$ has a subsequence converging to $K$, this implies that an arbitrarily small change of the feedback gain $K$ stabilizes the (unstable) system when $\tau=\tau_{\hat{n}}$ and we have a contradiction because the eigenvalues move continuously w.r.t. parameter changes, see Theorem 8 in the appendix.

Case 2: the sequence $\left\{\left\|K_{n}\right\|\right\}_{n} \geqslant 1$ is unbounded. First note that with an arbitrary feedback gain $K$ and for any $\omega>r_{\sigma}(A)$, the system $\dot{x}=A x+B K^{\mathrm{T}} x(t-\tau)$ has eigenvalues at $\pm \mathrm{j} \omega$ iff

$\operatorname{det}\left(I-(\mathrm{j} \omega I-A)^{-1} B K^{\mathrm{T}} \mathrm{e}^{-\mathrm{j} \omega \tau}\right)=0$,

meaning that the matrix $(\mathrm{j} \omega I-A)^{-1} B K^{\mathrm{T}} \mathrm{e}^{-\mathrm{j} \omega \tau}$ has an eigenvalue 1 . Since this is a rank-1 matrix, with its only possible nonzero eigenvalue equal to $\lambda_{n z}=$ $K^{\mathrm{T}}(\mathrm{j} \omega I-A)^{-1} B \mathrm{e}^{-\mathrm{j} \omega \tau}$, this is equivalent with $\left|\lambda_{n z}\right|=1$ and $\Im\left(\lambda_{n z}\right)=0$. Consequently, when $\left|K^{\mathrm{T}}(\mathrm{j} \omega I-A)^{-1} B\right|=1$, we have eigenvalues at $\pm \mathrm{j} \omega$ for a value of the delay $\tau$ satisfying $\tau \leqslant 2 \pi / \omega$. We will also use the fact that $\lim _{\omega \rightarrow \infty} K^{\mathrm{T}}(\mathrm{j} \omega I-A)^{-1} B=0$.

There exists a converging subsequence $\left\{F_{n}\right\}_{n} \geqslant 1$ of $\left\{K_{n} /\left\|K_{n}\right\|\right\}_{n \geqslant 1}$ and let its limit be $F$. Consider the parametrized curve in the complex plane, $\omega \in\left(r_{\sigma}(A), \infty\right) \rightarrow$ $F^{\mathrm{T}}(\mathrm{j} \omega I-A)^{-1} B$. It is impossible that this curve lies at zero for all $\omega>r_{\sigma}(A)$. Indeed when $\omega>r_{\sigma}(A)$, we can expand $(\mathrm{j} \omega I-A)^{-1}=\frac{1}{\mathrm{j} \omega} \sum_{k=0}^{\infty}(A / \mathrm{j} \omega)^{k}$. Hence

$$
\begin{aligned}
0= & F^{\mathrm{T}}(\mathrm{j} \omega I-A)^{-1} B \\
= & \frac{1}{\mathrm{j} \omega}\left[F^{\mathrm{T}} B+\frac{1}{\mathrm{j} \omega} F^{\mathrm{T}} A B+\frac{1}{(\mathrm{j} \omega)^{2}} F^{\mathrm{T}} A^{2} B \ldots\right] \\
& \forall \omega>r_{\sigma}(A)
\end{aligned}
$$

would imply that $F^{\mathrm{T}} A^{k} B=0, k \in \mathbb{N}$, and thus $F^{\mathrm{T}}[B A B \ldots$ $\left.A^{n-1} B\right]=0$. Because the pair $(A, B)$ is controllable, it follows that $F=0$ and we have a contradiction since $\|F\|=1$. 
As a consequence, $\exists \alpha>0$ and $\exists \bar{\omega}>r_{\sigma}(A)$ such that $\left|F^{\mathrm{T}}(\mathrm{j} \bar{\omega} I-A)^{-1} B\right|=\alpha$. Because $F_{n} \rightarrow F$ there exists an integer $N$ such that for $n \geqslant N, \frac{3}{2} \alpha \geqslant \mid F_{n}^{\mathrm{T}}(\mathrm{j} \bar{\omega} I-$ $A)^{-1} B \mid \geqslant \alpha / 2$. With a feedback gain $k F_{n}, k>0, n \geqslant N$, there are eigenvalues on the imaginary axis when $k \geqslant 2 / \alpha$ for a delay $\tau \leqslant 2 \pi / \bar{\omega}$, because then the curve $\omega \in\left(r_{\sigma}(A), \infty\right) \rightarrow k F_{n}^{\mathrm{T}}(\mathrm{j} \omega I-A)^{-1} B$ intersects the unit circle, and hence $\left|k F_{n}^{\mathrm{T}}(\mathrm{j} \omega I-A)^{-1} B\right|=1$, for some $\omega \geqslant \bar{\omega}$.

As a result, there is a subsequence of $\left\{K_{n}\right\}_{n \geqslant 0}$ which cannot asymptotically stabilize the system in delay intervals larger than $[0,2 \pi / \bar{\omega}]$ and we have a contradiction.

The next theorem illustrates an inherent difference between the ODE and the DDE case. When $\tau \neq 0$, the set of all stabilizing feedback gains is bounded, hence it is impossible to apply a high-gain approach and move the eigenvalues as far away as desired into the left half plane by increasing the controller gain.

Theorem 3. Suppose that a feedback $u=K^{\mathrm{T}} x$ asymptotically stabilizes the system $\dot{x}=A x+B u(t-\tau)$ for $\tau \in[0, r]$ with $r>0$. Then there exist constants $\gamma, \delta>0$ independent of $K$, such that $\|K\| \leqslant \gamma$ and, for each $\tau \in[0, r]$, $\inf _{K} \sup \Re(\lambda) \geqslant-\delta$.

Proof. Denote by $\mathscr{B}$ the unit ball in $\mathbb{R}^{n}$ and define $\bar{\omega}=\max \left(2 \pi / r, 2 r_{\sigma}(A)\right)$. For $K \in \mathscr{B}$, we have $\sup _{\omega \geqslant \bar{\omega}}\left|K^{\mathrm{T}}(\mathrm{j} \omega I-A)^{-1} B\right|>0$, because of the controllability of $(A, B)$, see the proof of Theorem 2 . From the compactness of $\mathscr{B}$ it follows that,

$$
\alpha \triangleq \inf _{K \in \mathscr{B}} \sup _{\omega \geqslant \bar{\omega}}\left|K^{\mathrm{T}}(\mathrm{j} \omega I-A)^{-1} B\right|>0 .
$$

Therefore, when the feedback gain $K$ satisfies $\|K\| \geqslant 1 / \alpha$, there will be eigenvalues on the imaginary axis for values of the delay smaller than $2 \pi / \bar{\omega} \leqslant r$, as follows from the arguments spelled out in the proof of Theorem 2. Hence, the family of all asymptotically stabilizing controls for the delay interval $[0, r]$ can be embedded in the set $\{K:\|K\| \leqslant 1 / \alpha \triangleq \gamma\}$, which proves the first part of the theorem. The second statement follows from the fact that $\sup \Re(\lambda)$ is continuous w.r.t. the components of $K$, see Theorem 8 in the appendix, and all stabilizing feedback gains belong to a compact set.

Remark 4. When $r \rightarrow 0$, we have $\gamma, \delta \rightarrow+\infty$, which links the DDE to the ODE situation.

\subsection{Methods based on prediction}

Existing methods based on finite spectrum assignment (Manitius \& Olbrot, 1979; Olbrot, 1978; Smith, 1957; Wang et al., 1999) avoid at first sight the conflict between the finite-dimensional controller parameter space and the infinite-dimensional closed loop system. They use an infinite-dimensional controller making the closed loop system finite dimensional. This can be done by counteracting the effect of the delay using a prediction of the state over a delay interval: for system (1), let $\hat{x}\left(t_{1}, t_{2}\right)$ be the prediction of $x$ at time $t_{2}$ based on its values for $t \leqslant t_{1}$. With the control law

$$
\begin{aligned}
u(t)= & K^{\mathrm{T}} \hat{x}(t, t+\tau)=K^{\mathrm{T}} \mathrm{e}^{A \tau} x(t) \\
& +K^{\mathrm{T}} \int_{0}^{\tau} \mathrm{e}^{A(\tau-\theta)} B u(t+\theta-\tau) \mathrm{d} \theta,
\end{aligned}
$$

the characteristic equation of the closed loop system is given by

$\operatorname{det}\left(\lambda I-A-B K^{\mathrm{T}}\right)=0$

and under the controllability assumption of the pair $(A, B)$, the $n$ closed loop eigenvalues can be assigned arbitrarily. However, in order to apply the control law (8), the integral term needs to be calculated on-line and in recent papers (Van Assche et al., 1999; Engelborghs et al., 2001) it is shown that the stability of closed loop system (1)-(8) might not be robust w.r.t. arbitrarily small implementation errors. The underlying reason is that the approximation error of the integral in (8), e.g. caused by an approximation with a finite sum, may form a noncompact perturbation of the semi-group associated with Eqs. (1)-(8). In the next subsection, we will show that for a one-dimensional example, the practical stabilizability properties with a control law of form (8) are qualitatively the same as with the simple state feedback $u=K^{\mathrm{T}} x$.

\subsection{The two approaches applied to a theoretical example}

We investigate the stabilization of the system,

$\dot{x}=a x+u(t-\tau), \quad x \in \mathbb{R}$.

Using the control law $u=k x$ we obtain the closed loop system

$\dot{x}=a x+k x(t-\tau)$,

which is also analyzed in Cooke and Grossman (1982), Dugard and Verriest (1998) and Kolmanovskii and Myshkis (1999). Fig. 1 shows the stability regions in the parameter space $(a, k)$. The boundary of these regions can be calculated by substituting $\lambda=\mathrm{j} \omega, \omega \in \mathbb{R}$ in the characteristic equation $\lambda-a-k \mathrm{e}^{-\lambda \tau}=0$. The system is asymptotically stable for all values of the delay when $a<0$ and $-|a| \leqslant k<|a|$. Note that when the uncontrolled system is unstable $(a>0)$, delay independent stability is not possible (Theorem 1 ). When only asymptotic stability for all delays $\tau \leqslant r$ is required, the stability region extends to the curve through the 


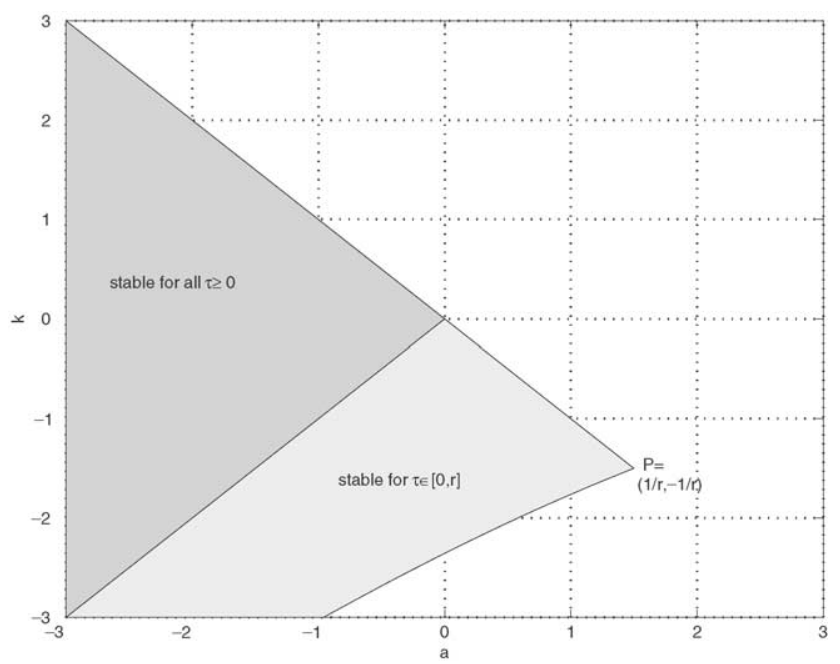

Fig. 1. Stability regions of $\dot{x}=a x+k x(t-\tau)$.

point $P=(1 / r,-1 / r)$, analytically given by

$a=\frac{\omega}{\tan (\omega r)}, \quad k=-\frac{\omega}{\sin (\omega r)}, \quad \omega r \in[0, \pi)$,

see Fig. 1. Hence, it is impossible to stabilize the system for all $\tau \in[0, r]$ when $r>1 / a$, whatever the value of the feedback gain $k$ (Theorem 2 ). Note that the stabilizability condition is given by $a r<1$, which can be interpreted as a tradeoff between the instability of the uncontrolled system $(a)$ and the destabilizing effect of the delay $(r)$. One can show ${ }^{1}$ that for a particular value of the delay $\tau$, a necessary and sufficient condition for asymptotic stabilizability of (9) is also given by,

$a \tau<1$.

For $a>0$ and $\tau<1 / a$, the stabilizing values of $k$ lie in a compact interval (Theorem 3 ) and a right upper bound on the spectrum can never reach $-\infty$. A simple calculation shows that the optimal stabilizing feedback, i.e. the value of $k$ which minimizes $\sup \Re(\lambda)$, is given by $k=-(1 / \tau) \mathrm{e}^{a \tau-1}$ resulting in $c_{1}=\inf _{k} \sup (\Re(\lambda))=a-(1 / \tau)$.

In Engelborghs et al. (2001), one considers the stabilization of system (9) with $a=1$ using the feedback $u=-\left(1-\lambda_{d}\right)\left(\mathrm{e}^{\tau} x+\int_{0}^{\tau} \mathrm{e}^{\tau-\theta} u(t+\theta-\tau) \mathrm{d} \theta\right)$, yielding one closed loop eigenvalue at $\lambda_{d}$. The robustness of the controller is investigated when the integral term is implemented with a numerical quadrature rule, e.g. the trapezoidal rule. Since placing $\lambda_{d}$ far away into the left half plane increases the destabilizing influence of the implementation error, there also exists an optimal position of $\lambda_{d}$. In Fig. 2, $c_{2}=\inf _{\lambda_{d}} \sup \Re(\lambda)$ is depicted as a function of $\tau$ (solid line), taken into account the influence of arbitrarily small implementation errors. For $\tau>1.293$, the system cannot be stabilized. The dashed line shows the

\footnotetext{
${ }^{1}$ This follows from $\mathrm{d} \Re(\lambda) / \mathrm{d} \tau \geqslant 0$ for eigenvalues on the imaginary axis, see Cooke and Grossman (1982).
}

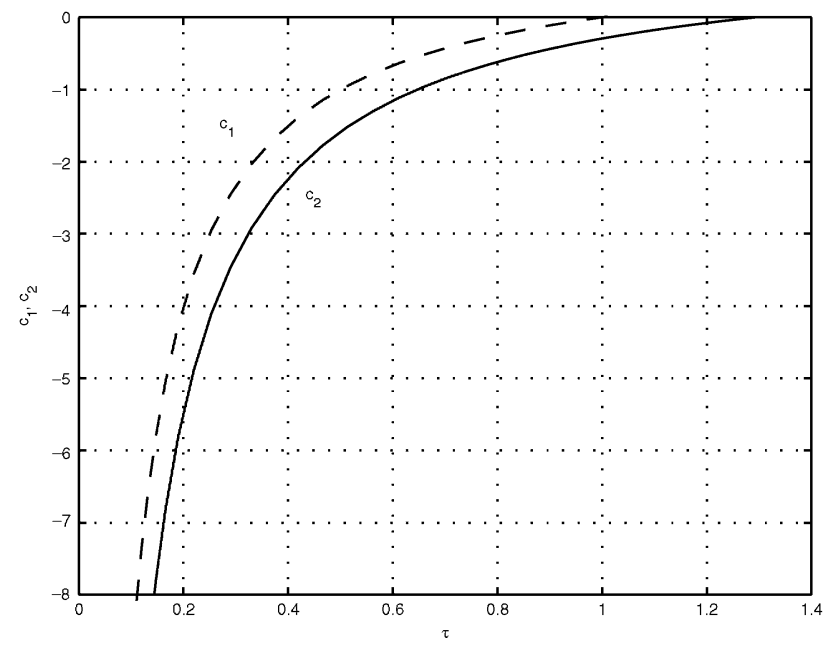

Fig. 2. Stabilizability limits of the system $\dot{x}=x+u(t-\tau)$.

maximal exponential decay rate in the case of pure state feedback, $c_{1}=\inf _{k} \sup \Re(\lambda)$. This figure makes clear that from a practical point of view, both methods are comparable. Using the techniques of the same paper (Engelborghs et al., 2001) one can show that for system (9) the (practical) stabilizability condition in case of finite spectrum assignment is given by

$a \tau<1.293$,

only slightly better than (11). In Section 6, we will show that with a modification of the continuous pole placement method, we are even able to improve this stabilizability condition.

\section{The continuous pole placement method}

\subsection{Description of the algorithm}

The idea behind this stabilization method is to shift the unstable eigenvalues to the left half plane in a quasi-continuous way by applying small changes to the feedback gain, while monitoring the other eigenvalues with a large real part. It is based on the continuity of the eigenvalues w.r.t. the components of the feedback gain (Theorem 8 in the appendix). The basic algorithm is as follows:

Algorithm 1. The continuous pole placement method

$A$. Initialize $m=1$.

$B$. Compute the rightmost eigenvalues for the nominal delay $\tau$.

$C$. Compute the sensitivity of the $m$ rightmost eigenvalues w.r.t. changes in the feedback gain $K$.

$D$. Move the $m$ rightmost eigenvalues in the direction of the left half plane by applying a small change to the feedback gain $K$, using the computed sensitivities. 
$E$. Monitor the rightmost uncontrolled eigenvalues. If necessary, increase the number of controlled eigenvalues, $m$. Stop when stability is reached or when the available degrees of freedom in the controller do not allow to further reduce $\sup \Re(\lambda)$. In the other case, go to step $B$.

We will now describe the different steps of the algorithm in more detail.

\subsubsection{Computation of the rightmost eigenvalues}

In Engelborghs and Roose (1999) a method is proposed which automatically computes the rightmost eigenvalues of the characteristic equation. First, a discretization is obtained of the time integration operator of the linear or linearized system of DDEs, whose eigenvalues are exponential transforms of the roots of the characteristic equation. Then, selected eigenvalues of the resulting large matrix are computed. The effect of discretization on stability using linear multi-step methods is well understood, see, e.g. Hong-Jiong and Jiao-Xun (1996) and $\mathrm{Hu}, \mathrm{Hu}$, and Liu (1997). A steplength heuristic is used to ensure that all eigenvalues of interest are accurately approximated by the discretization. Accuracy can be increased by employing a Newton iteration on the characteristic equation using the approximate eigenvalues as starting values. This method has been implemented in the Matlab package DDE-BIFTOOL (Engelborghs, 2000).

\subsubsection{Sensitivity of eigenvalues with respect to the feedback gain $K$}

When $\lambda_{i}$ is a solution of the characteristic equation and $v_{i} \mathrm{e}^{\lambda \theta}, \theta \in[-\tau, 0]$, its corresponding eigenfunction, we have

$\left(\lambda_{i} I-A-B K^{\mathrm{T}} \mathrm{e}^{-\lambda_{i} \tau}\right) v_{i}=0$,

$n\left(v_{i}\right)=0$,

where $n\left(v_{i}\right)$ is a normalizing condition. Differentiating this equation w.r.t. a component $k_{j}$ of $K$, we obtain a linear system of equations in the unknowns $\partial \lambda_{i} / \partial k_{j}$ and $\partial v_{i} / \partial k_{j}$ :

$$
\begin{aligned}
& {\left[\begin{array}{cc}
\lambda_{i} I-A-B K^{\mathrm{T}} \mathrm{e}^{-\lambda_{i} \tau} & \left(I+B K^{\mathrm{T}} \tau \mathrm{e}^{-\lambda_{i} \tau}\right) v_{i} \\
\frac{\mathrm{d} n^{\mathrm{T}}}{\mathrm{d} v_{i}} & 0
\end{array}\right]\left[\begin{array}{c}
\frac{\partial v_{i}}{\partial k_{j}} \\
\frac{\partial \lambda_{i}}{\partial k_{j}}
\end{array}\right]} \\
& =\left[\begin{array}{c}
B v_{i}^{\mathrm{T}} e_{j} \mathrm{e}^{-\lambda_{i} \tau} \\
0
\end{array}\right]
\end{aligned}
$$

with $e_{j} \in \mathbb{R}^{n \times 1}$ the $j$ th unity vector.
When the system is in the control canonical form (5), one can compute directly from (7),

$$
\frac{\partial \lambda_{i}}{\partial k_{j}}=-\frac{\mathrm{e}^{-\lambda_{i} \tau} \lambda_{i}^{j-1}}{\mathrm{~d} H / \mathrm{d} \lambda_{i}} .
$$

This result is useful for the derivation of theoretical properties of the method, but our implementation is based on (13), since Eq. (7) is not practical from a numerical point of view.

\subsubsection{Continuation of eigenvalues as a function of the feedback gain $K$}

Assume that $m \leqslant n$ eigenvalues $\lambda_{1}, \ldots, \lambda_{m}$ are controlled. When the 'sensitivity' matrix $S_{m}$, defined by

$S_{m}=\left[s_{i, j}\right] \in \mathbb{R}^{m \times n} \quad$ where $s_{i, j}=\frac{\partial \lambda_{i}}{\partial k_{j}}$,

is of rank $m$ and the desired (small) displacement of the controlled eigenvalues is given by $\Delta \Lambda_{m}^{d}=\left[\Delta \lambda_{1}^{d} \ldots \Delta \lambda_{m}^{d}\right]^{\mathrm{T}}$, one can compute a change $\Delta K$ for $K$ such that

$S_{m} \Delta K=\Delta \Lambda_{m}^{d}$.

When $m<n$ this equation has infinitely many solutions. One possibility to determine a unique solution consists of controlling the $m$ eigenvalues using only $m$ selected components of $K$ and hence taking $n-m$ components of $\Delta K$ equal to zero. Another possibility, which we will motivate later, consists of taking the solution with $\|\Delta K\|$ minimal. This solution is given by

$\Delta K=S_{m}^{\dagger} \Delta \Lambda_{m}^{d}$,

where $S_{m}^{\dagger}$ is the Moore-Penrose inverse of $S_{m}$, see Ben-Israel and Greville (1974, Chap. 3).

A physical constraint on the feedback gain is imposed by the fact that its components must be real, but this is assured by taking the components of $\Delta \Lambda_{m}^{d}$ in complex conjugate pairs.

With the new feedback gain $K+\Delta K$, the displacement of the controlled eigenvalues will generically not be equal to $\Delta \Lambda_{m}^{d}$, since Eq. (17) is based on linearization, and some correction is needed. However, since the eigenvalues and eigenfunctions are continuous w.r.t. parameter changes, with for instance the predictor

$\lambda_{i}^{(p)}=\lambda_{i}+\Delta \lambda_{i}^{d}, \quad v_{i}^{(p)}=\sum_{j=1}^{n} \frac{\partial v_{i}}{\partial k_{j}} \Delta k_{j}, \quad i=1, m$

only a few Newton iterations on Eq. (13) are needed when $\Delta K_{m}$ is sufficiently small. Since it is also desirable to have the eigenvalues for the new feedback gain close to their predictions, $(18)$ is generally a good choice for the solution of (17).

The $n$ components of the controller gain allow to control both real and imaginary parts of at most $n$ eigenvalues, as illustrated by (17), or, when stabilization 

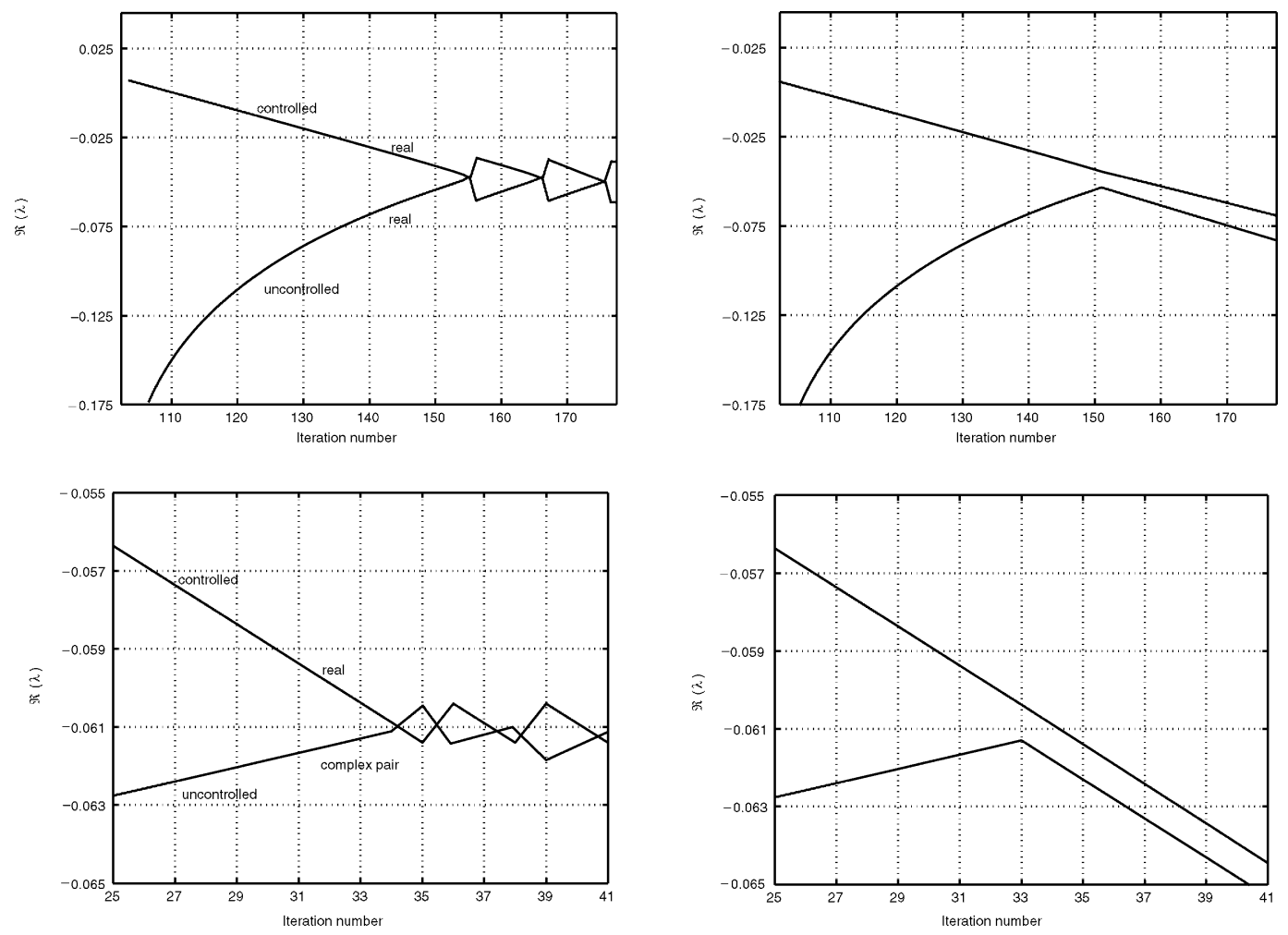

Fig. 3. Two typical situations where it is necessary to extend the number of controlled eigenvalues for a further reduction of sup $\Re(\lambda)$.

is of main concern, only the real part of possibly more than $n$ eigenvalues. For instance, with $n=2$ degrees of freedom in the controller, one can control either two real eigenvalues, or one real eigenvalue and the real part of a complex pair, or the real parts of two complex pairs. For the latter case, note that

$\frac{\partial \mathfrak{R}\left(\lambda_{i}\right)}{\partial k_{j}}=\mathfrak{R}\left(\frac{\partial \lambda_{i}}{\partial k_{j}}\right)$.

This approach is applied to all examples of the paper, combined with an adaptation of all controller parameters in an iteration step, as in (18). This leads to the modified adaptation formula for the feedback gain,

$\Delta K=\left(\Re\left(S_{m}\right)\right)^{\dagger} \Delta \mathfrak{R}\left(\Lambda_{m}^{d}\right)$,

where $\Delta \mathfrak{R}\left(\Lambda_{m}^{d}\right)$ is the desired displacement of the real parts of the controlled eigenvalues.

\subsubsection{Increasing the number of controlled eigenvalues}

In the examples of the paper, where the focus lies on stabilization, we only control the real parts of the eigenvalues. We start by shifting the dominant real eigenvalue or by reducing the real part of the dominant complex pair. In this way control is lost over the other eigenvalues and whenever an interaction occurs, the number of controlled eigenvalues, $m$, generally needs to be increased to further reduce $\sup \Re(\lambda)$. This will now be illustrated.
In Fig. 3, we display two typical situations, where the real part of only the rightmost eigenvalue is controlled and the number of controlled eigenvalues must be increased when it interacts with other eigenvalues. In the upper part the situation is shown where two real eigenvalues interact. When only the dominant eigenvalue is controlled, no further reduction of $\sup \Re(\lambda)$ is possible after the interaction (left): when the two eigenvalues coincide, they first transform to a complex conjugate pair which does not move to the left in the complex plane by the next parameter change (we control only its real part), but immediately splits up again into two real eigenvalues, and then the whole process repeats itself. By controlling the two real eigenvalues from iteration number 150 on, a further reduction of $\sup \Re(\lambda)$ is possible (right). In the lower part of Fig. 3, a real eigenvalue interacts with a complex pair of eigenvalues. A decrease of the real eigenvalue leads to an increase of the real part of the complex pair and vice versa (left). This problem can be avoided by controlling both the real eigenvalue and the real part of the complex pair (right).

As will be illustrated with the numerical examples in the paper, a special situation also occurs when a complex pair, whose real part is controlled, splits up into two real eigenvalues. This is possible because its imaginary part is not controlled and may tend to zero during the stabilization procedure. After the splitting both real eigenvalues need to be controlled. 
In the current version of the algorithm, the number of controlled eigenvalues is manually increased, whenever stagnation of $\sup \Re(\lambda)$ or a splitting of a complex pair occurs. The method breaks down when no further reduction of $\sup \Re(\lambda)$ is possible, i.e. when the available degrees of freedom in the controller $(n)$ is not sufficient to control the real parts of all dominant eigenvalues. This can happen when $n+1$ real parts need to be controlled to reduce $\sup \mathfrak{R}(\lambda)$ or when the matrix $\mathfrak{R}\left(S_{m}\right)$ in (19) has a rank strictly smaller than $m$.

\subsection{Theoretical properties}

In each step of the continuous pole placement algorithm, the sensitivity matrix $S_{m}$ must be of rank $m$, if full control over the changes of the $m$ eigenvalues is desired. The next theorem states that when both real and imaginary parts of $n$ eigenvalues of multiplicity 1 are controlled, the sensitivity matrix $S_{n}$ is always regular.

Theorem 5. If $\lambda_{1}, \ldots, \lambda_{n}$ are single eigenvalues, then the sensitivity matrix $S_{n}$ is regular.

Proof. Since the pair $(A, B)$ is controllable, the system can be transformed into the control canonical form (5)-(7). Using (15), we obtain the following sensitivity matrix:

$S_{n}=\left[\begin{array}{ccc}\frac{\mathrm{e}^{-\lambda_{1} \tau}}{\mathrm{d} H / \mathrm{d} \lambda_{1}} & & \\ & \ddots & \\ & & \frac{\mathrm{e}^{-\lambda_{n} \tau}}{\mathrm{d} H / \mathrm{d} \lambda_{n}}\end{array}\right]\left[\begin{array}{cccc}1 & \lambda_{1} & \ldots & \lambda_{1}^{n-1} \\ 1 & \lambda_{2} & \ldots & \lambda_{2}^{n-1} \\ \vdots & \vdots & \ldots & \vdots \\ 1 & \lambda_{n} & \ldots & \lambda_{n}^{n-1}\end{array}\right]$.

This matrix is regular if no eigenvalues coincide, since $\mathrm{d} H / \mathrm{d} \lambda_{i} \neq 0$ and the Vandermonde matrix is regular.

When two eigenvalues are close to each other, the sensitivity w.r.t. changes in the feedback gain is large, as shown in the next theorem. This result is related to the fact that large qualitative changes in the spectral picture can occur when eigenvalues coincide.

Theorem 6. When two eigenvalues are brought together, the norm of the sensitivity matrix $S_{n}$ becomes arbitrarily large.

Proof. Start from (20) and consider the asymptotic case where $\lambda_{j} \rightarrow \lambda_{i}$ while the other eigenvalues are kept fixed. With the explicit expression for the determinant of a Vandermonde matrix, we have

$\lim _{\lambda_{j} \rightarrow \lambda_{i}} \operatorname{det}\left(S_{n}\right)=\mathrm{e}^{-2 \lambda_{i} \tau} \prod_{k=1, k \neq i, k \neq j}^{n}\left(\frac{\mathrm{e}^{-\lambda_{k} \tau}}{\mathrm{d} H / \mathrm{d} \lambda_{k}}\right)$

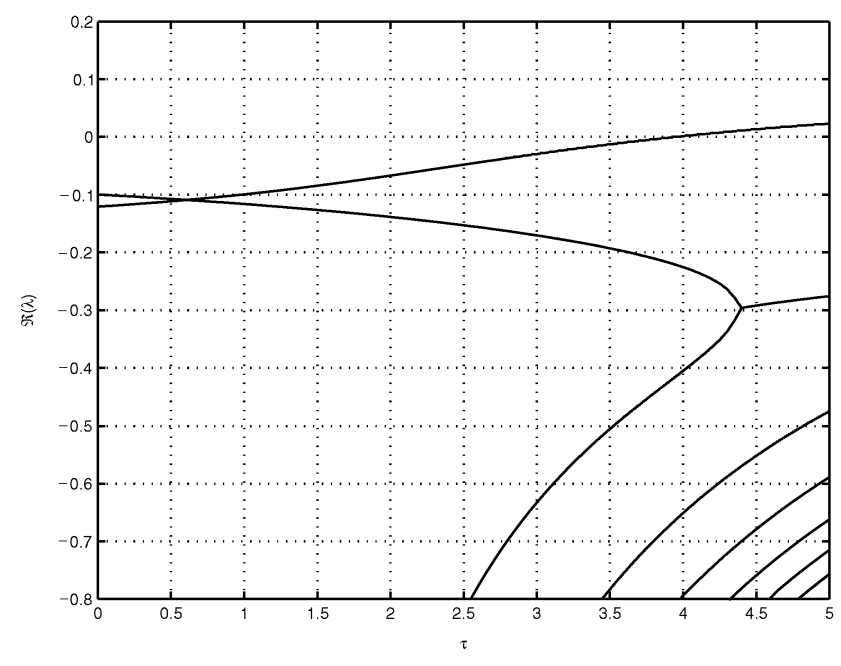

Fig. 4. Rightmost eigenvalues of the system (21)-(22) as a function of the delay, $K=\left[\begin{array}{lll}0.719 & 1.04 & 1.29\end{array}\right]^{\mathrm{T}}$.

$$
\lim _{\lambda_{j} \rightarrow \lambda_{i}}\left(\frac{1}{\mathrm{~d} H / \mathrm{d} \lambda_{i} \mathrm{~d} H / \mathrm{d} \lambda_{j}} \prod_{l>k=1}^{n}\left(\lambda_{l}-\lambda_{k}\right)\right)
$$

and $\operatorname{det}\left(S_{n}\right) \rightarrow \infty$ implies $\left\|S_{n}\right\| \rightarrow \infty$ for any induced norm $\|$.$\| .$

When only the real parts of eigenvalues are controlled, it is recommended to keep the real parts separated in order to avoid highly sensitive sensitivity matrices leading to numerical problems. This is illustrated with the example in the next section.

\section{Example}

We illustrate the continuous pole placement method and its theoretical properties by means of the system

$\dot{x}=A x+B u(t-\tau), \quad u=K^{\mathrm{T}} x$,

where

$A=\left[\begin{array}{ccc}-0.08 & -0.03 & 0.2 \\ 0.2 & -0.04 & -0.005 \\ -0.06 & 0.2 & -0.07\end{array}\right], \quad B=\left[\begin{array}{c}-0.1 \\ -0.2 \\ 0.1\end{array}\right]$,

$\tau=5$.

The uncontrolled system is unstable $(\sup \Re(\lambda)=$ 0.108 ) and with the feedback $u=K^{\mathrm{T}} x$ where $K=$ $\left[\begin{array}{lll}0.719 & 1.04 & 1.29\end{array}\right]^{\mathrm{T}}$, the rightmost eigenvalues are shown in Fig. 4. Calculations were made using the Matlab package DDE-BIFTOOL (Engelborghs, 2000). Note that as $\tau \rightarrow 0+$, the three dominant eigenvalues converge to the ODE eigenvalues while the other move off to $-\infty$. 


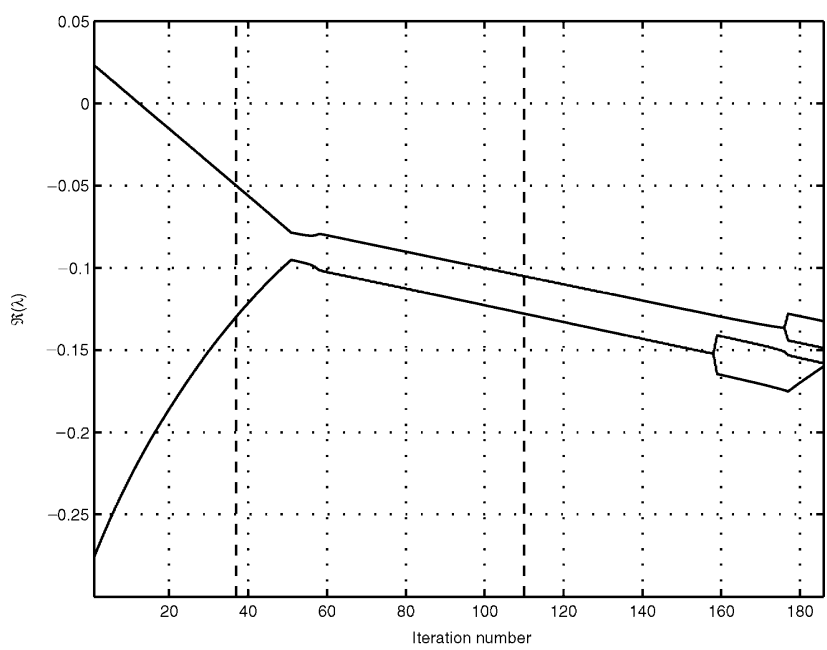

Fig. 5. Real parts of the the rightmost eigenvalues of (21)-(22) with $\tau=5$ as a function of the iterations of the continuous pole placement algorithm. For values of the feedback gain at iterations 37, 110 (indicated by the dashed lines), and its value in the optimum, the rightmost eigenvalues are continued as a function of the delay in Fig. 7.

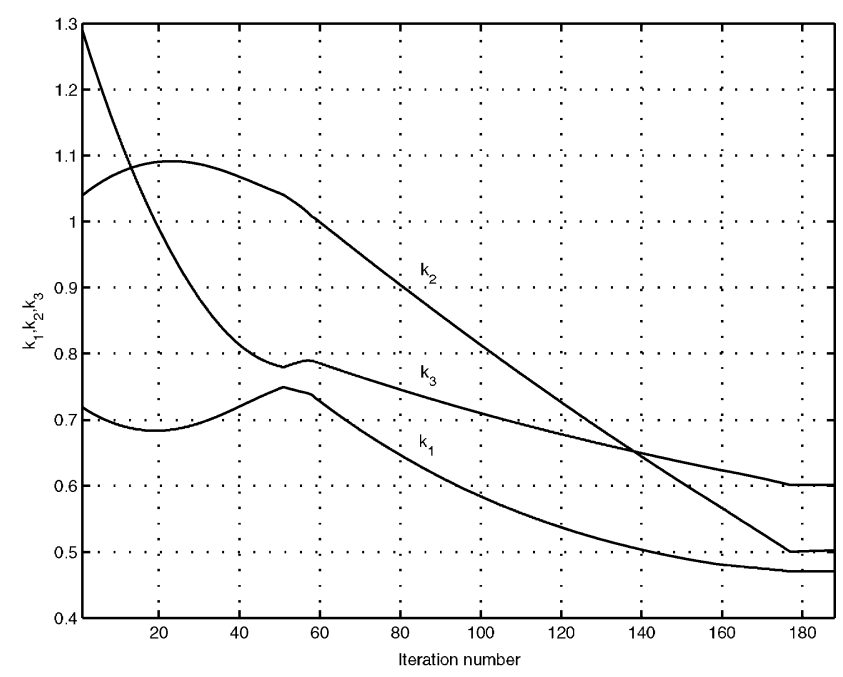

Fig. 6. Feedback gain $K=\left[\begin{array}{lll}k_{1} & k_{2} & k_{3}\end{array}\right]^{\mathrm{T}}$ as a function of the iterations of the continuous pole placement algorithm applied to (21)-(22).

Although the particular control law achieves stability for $\tau=0$, the system is unstable for the nominal delay $\tau=5$ and we now describe its stabilization with the continuous pole placement method.

In Fig. 5, the rightmost eigenvalues are shown as a function of the number of iterations taken in Algorithm 1. The corresponding values of the feedback gain $K=$ $\left[\begin{array}{lll}k_{1} & k_{2} & k_{3}\end{array}\right]^{\mathrm{T}}$ are displayed in Fig. 6. First we only reduce the real part of the dominant pair of complex conjugate eigenvalues, which is already sufficient to achieve stabilization. Meanwhile the real part of an uncontrolled pair grows, and after 50 iterations we reduce the real part of the two complex pairs of eigenvalues. In this way, we loose control over the imaginary parts and because we want to avoid coinciding eigenvalues, which cause numerical problems (Theorem 6), we keep the real parts separated. However, around iteration 58, the eigenvalues are close and the effect of a high sensitivity w.r.t. changes in the feedback gain is visible. At iteration 158, a complex pair splits into two real eigenvalues and all components of the feedback gain $K$ are used to reduce these real eigenvalues and the real part of the dominant pair. From iteration 176 on, where also this pair splits, we only control the three dominant real eigenvalues and around iteration 190 the method breaks down. At this point we are close to the minimum of $\sup \Re(\lambda)$ characterized by four coinciding rightmost eigenvalues at $\lambda=-0.150$. The corresponding feedback gain is given by $K=\left[\begin{array}{lll}0.471 & 0.504 & 0.602\end{array}\right]^{\mathrm{T}}$.

In Fig. 7 the rightmost eigenvalues are displayed as a function of the delay at iterations 37,110 , and in the optimum. This figure illustrates how the real parts of the dominant eigenvalues evolve towards the minimum for the nominal delay, characterized by coinciding eigenvalues and the resulting high sensitivity w.r.t. parameter changes (including the delay). This high sensitivity around the optimum is a property of the system. Our experience indicates that for this type of control problems, coinciding eigenvalues are a general characteristic of the global optimum. Another illustration can be found in Michiels and Roose (2001), where all possible configurations of the rightmost eigenvalues in the global minimum of $\sup \mathfrak{R}(\lambda)$ were given for 2D systems. These were all characterized by (some) eigenvalues with multiplicity larger than one. In our example the high sensitivity w.r.t. delay changes for the final feedback gain is caused by the fact that we executed Algorithm 1 until we reached the minimum. However, from a practical point of view, where also the robustness aspect is important, this is not needed. For instance, the feedback gain obtained at iteration number 37, see Fig. 7, already achieves stability. The exponential decay rate of the closed loop solution is smaller than for the optimum, yet less sensitive to delay changes.

\section{Observer based controller}

We now assume that not the full state $x$, but only an output $y=C^{\mathrm{T}} x \in \mathbb{R}$, is available for measurement, with $(C, A)$ observable. Note that in this case the system can be equivalently described by the transfer function

$G_{\tau}(s)=G(s) \mathrm{e}^{-s \tau}$,

with $G(s)=C^{\mathrm{T}}(s I-A)^{-1} B$, and that any system of the form

$\dot{x}=A x+B u\left(t-\tau_{1}\right), \quad y=C^{\mathrm{T}} x\left(t-\tau_{2}\right), \quad \tau_{1}+\tau_{2}=\tau$, 

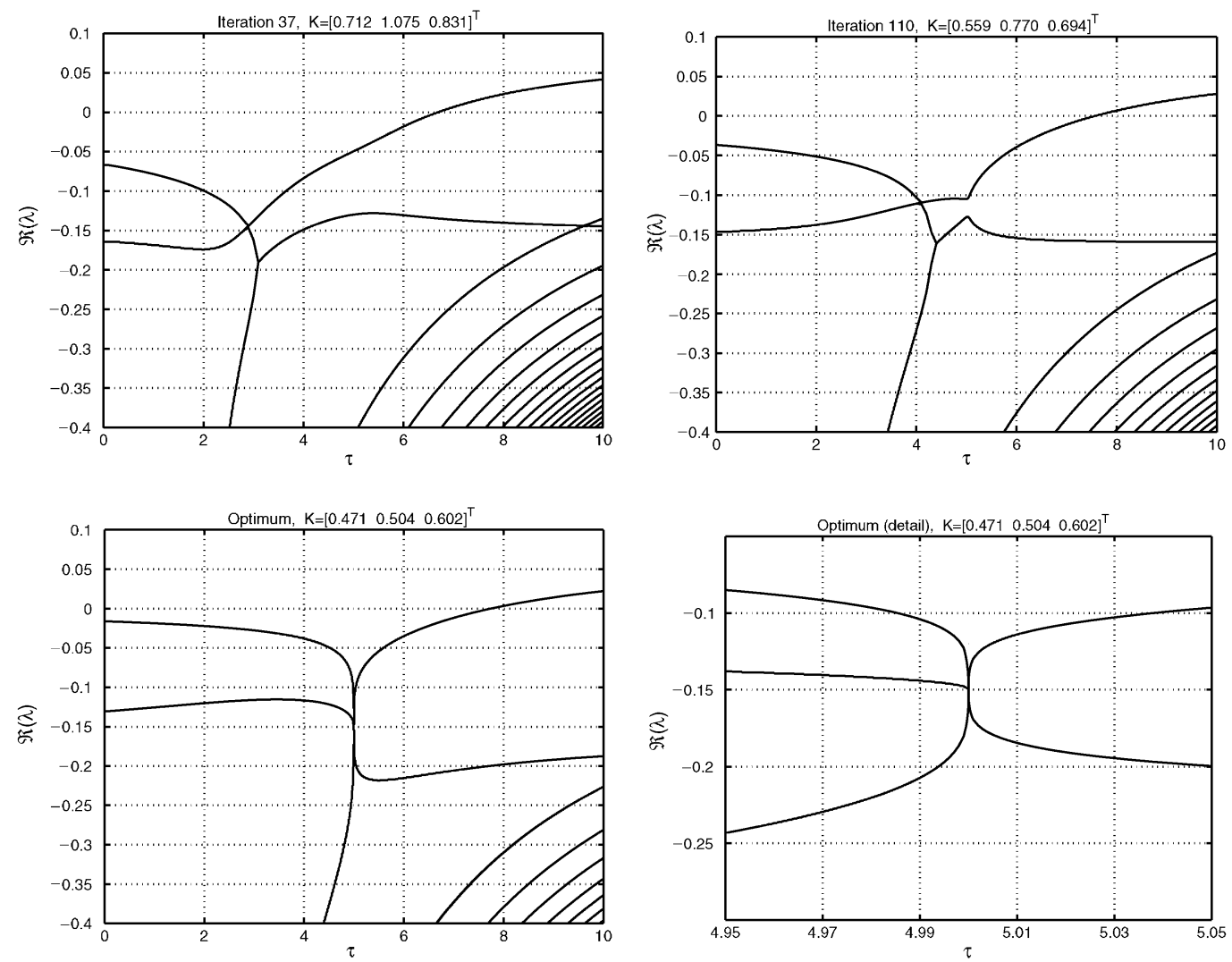

Fig. 7. Rightmost eigenvalues of (21)-(22) as a function of the delay, for values of $K$ at iteration 37 (upper left), iteration 110 (upper

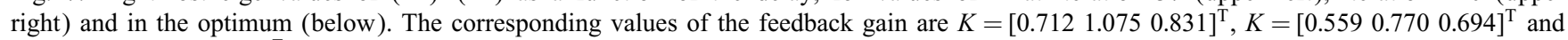
$K=\left[\begin{array}{lll}0.471 & 0.504 & 0.602\end{array}\right]^{\mathrm{T}}$.

is a realization of (23). In order to apply the continuous pole placement method, we construct an observer for (24),

$\dot{\hat{x}}=A \hat{x}+B u\left(t-\tau_{1}\right)+L\left(C^{\mathrm{T}} \hat{x}\left(t-\tau_{2}\right)-y\right)$,

with $L \in \mathbb{R}^{n \times 1}$ the observer gain, and apply the feedback $u=K^{\mathrm{T}} \hat{x}(t)$. With $e=x-\hat{x}$ the observer error, we obtain

$\dot{x}=A x+B K^{\mathrm{T}} x\left(t-\tau_{1}\right)-B K^{\mathrm{T}} e\left(t-\tau_{1}\right)$,

$\dot{e}=A e+L C^{\mathrm{T}} e\left(t-\tau_{2}\right)$.

Since in the characteristic equation of the closed loop system (25),

$$
\begin{gathered}
\operatorname{det}\left(\lambda I-\left[\begin{array}{ll}
A & 0 \\
0 & A
\end{array}\right]-\left[\begin{array}{cc}
B K^{\mathrm{T}} & -B K^{\mathrm{T}} \\
0 & 0
\end{array}\right] \mathrm{e}^{-\lambda \tau_{1}}\right. \\
\left.-\left[\begin{array}{cc}
0 & 0 \\
0 & L C^{\mathrm{T}}
\end{array}\right] \mathrm{e}^{-\lambda \tau_{2}}\right)=0,
\end{gathered}
$$

all matrices are block-triangular, the separation principle is valid and the closed loop eigenvalues consist of the solutions of $\operatorname{det}\left(\lambda I-A-B K^{\mathrm{T}} \mathrm{e}^{-\lambda \tau_{1}}\right)=0$, i.e. the controller eigenvalues, and of $\operatorname{det}\left(\lambda I-A-L C^{\mathrm{T}} \mathrm{e}^{-\lambda \tau_{2}}\right)=$ $\operatorname{det}\left(\lambda I-A^{\mathrm{T}}-C L^{\mathrm{T}} \mathrm{e}^{-\lambda \tau_{2}}\right)=0$, i.e. the observer eigenvalues. Hence to achieve stability we apply the continuous pole placement twice, to the systems $\left(A, B, \tau_{1}\right)$ and $\left(A^{\mathrm{T}}, C, \tau_{2}\right)$.

In the construction of realization (24), we are free to distribute the delay over input and output. This additional degree of freedom can be used to obtain better performance of plant and observer. It also allows to extend the class of systems which can be stabilized with the continuous pole placement method. To see this, reconsider system (9) with output $y=x$. Although the full state is available for feedback, it will be useful to construct an observer. The transfer function is given by $G_{\tau}(s)=$ $\mathrm{e}^{-s \tau} / s-a$ and we construct an observer based on the realization,

$\dot{x}=a x+u\left(t-\tau_{1}\right), \quad y=x\left(t-\tau_{2}\right), \quad \tau_{1}+\tau_{2}=\tau$.

With the observer $\dot{\hat{x}}=a \hat{x}+u\left(t-\tau_{1}\right)+l\left(\hat{x}\left(t-\tau_{2}\right)-y\right)$ and the controller $u=k \tilde{x}$, the closed loop eigenvalues coincide with the eigenvalues of the systems,

$\dot{x}=a x+k x\left(t-\tau_{1}\right), \quad \dot{e}=a e+l e\left(t-\tau_{2}\right)$. 

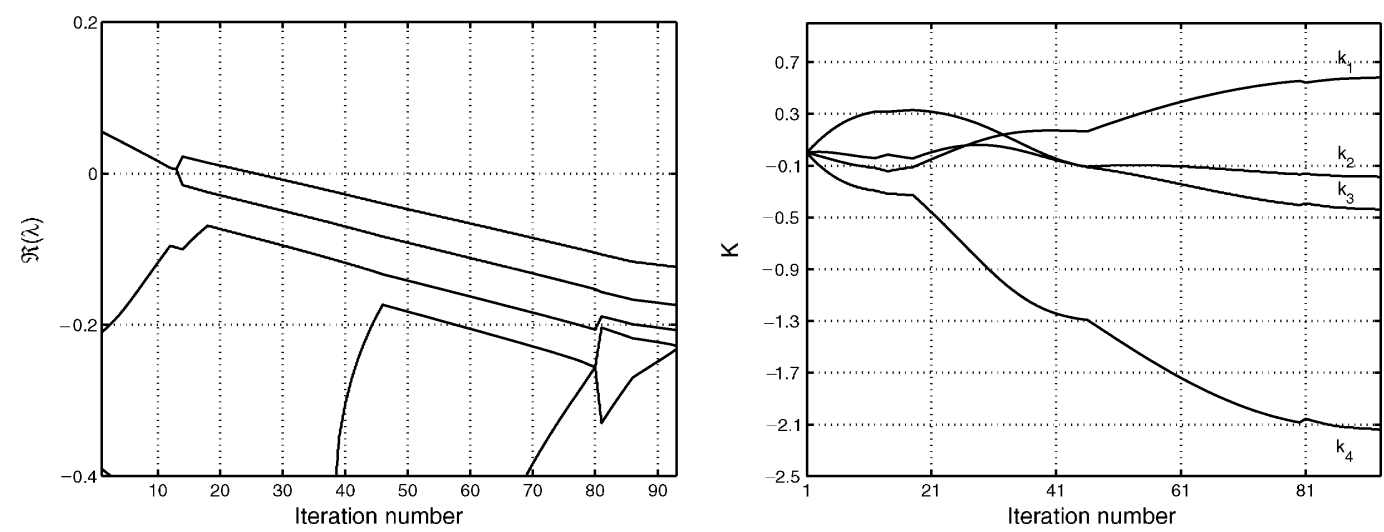

Fig. 8. Rightmost eigenvalues of (28) (left) and components of the feedback gain $K=\left[\begin{array}{llll}k_{1} & k_{2} & k_{3} & k_{4}\end{array}\right]^{\mathrm{T}}$ (right) as a function of the iterations of the continuous pole placement algorithm.

Since these equations are of form (10), the optimal stabilizing controller (i.e. values of $k$ and $l$ which minimize $\sup \Re(\lambda)$ ) results in,

$\sup \Re(\lambda)=\max \left(a-\frac{1}{\tau_{1}}, a-\frac{1}{\tau_{2}}\right)$,

and the best stabilizability results are obtained when distributing the delay equally over input and output. Hence asymptotic stability can be achieved iff

$a \tau<2$,

which is twice as good as (11), valid for pure state feedback, and better than (12), the 'practical' condition in case of finite spectrum assignment.

\section{Extension}

The continuous pole placement method can be considered as a natural generalization of the classical pole placement algorithm to the input delay case. Since it is based on the continuous dependence of the rightmost eigenvalues on the controller parameters and because the algorithms described in Section 4.1.1 can also deal with equations with several discrete delays, it can easily be extended to more general types of linear delay equations involving delays in both state and control variables, and multiple feedbacks. Although theoretical stabilizability properties and convergence results, such as Theorem 5, are generally not valid and depend on the structure of the system under consideration, we will briefly illustrate with some examples the effectiveness of the method in solving more complicated stabilization problems.

As a first example consider the following system,

$\dot{x}=A_{1} x+A_{2} x\left(t-\tau_{1}\right)+B_{1} u\left(t-\tau_{2}\right)+B_{2} u\left(t-\tau_{3}\right)$,

$u=K^{\mathrm{T}} x$, where

$\begin{aligned} A_{1} & =\left[\begin{array}{cccc}0.1 & -0.5 & -0.3 & 0.3 \\ 0 & 0 & 0.2 & -0.2 \\ 0 & 0 & -0.1 & 0 \\ 0 & 0.4 & 0.3 & -0.4\end{array}\right], \\ A_{2} & =\left[\begin{array}{cccc}-0.2 & 0.3 & 0.2 & -0.5 \\ 0 & -0.2 & -0.2 & 0.2 \\ -0.1 & 0.1 & 0.1 & 0.1 \\ 0.1 & -0.6 & -0.6 & 0\end{array}\right],\end{aligned}$

$B_{1}=\left[\begin{array}{c}0 \\ -0.1 \\ 0 \\ 0.1\end{array}\right], \quad B_{2}=\left[\begin{array}{c}-0.3 \\ 0 \\ 0 \\ 0\end{array}\right]$,

$\tau_{1}=2, \quad \tau_{2}=1, \quad \tau_{3}=3$,

and a stabilizing feedback gain $K=\left[\begin{array}{llll}k_{1} & k_{2} & k_{3} & k_{4}\end{array}\right]^{\mathrm{T}}$ needs to be determined. In Fig. 8, the rightmost eigenvalues of (28) are shown as a function of the iterations of the continuous pole placement algorithm, as well as the components of the feedback gain. The initial value of the feedback gain is $\left[\begin{array}{llll}0 & 0 & 0 & 0\end{array}\right]^{\mathrm{T}}$. We have four control parameters and the method converges towards an optimum characterized by five coinciding real eigenvalues (in the last iteration step we already have a good approximation when taking into account the high sensitivity around the optimum).

Now consider the case where the two inputs in (28) are independent,

$\dot{x}=A_{1} x+A_{2} x\left(t-\tau_{1}\right)+B_{1} u_{1}\left(t-\tau_{2}\right)+B_{2} u_{2}\left(u-\tau_{3}\right)$,

$u_{1}=K_{1}^{\mathrm{T}} x, \quad u_{2}=K_{2}^{\mathrm{T}} x$,

with the system parameters given by (29). In Fig. 9, iterations of the continuous pole placement algorithm are 

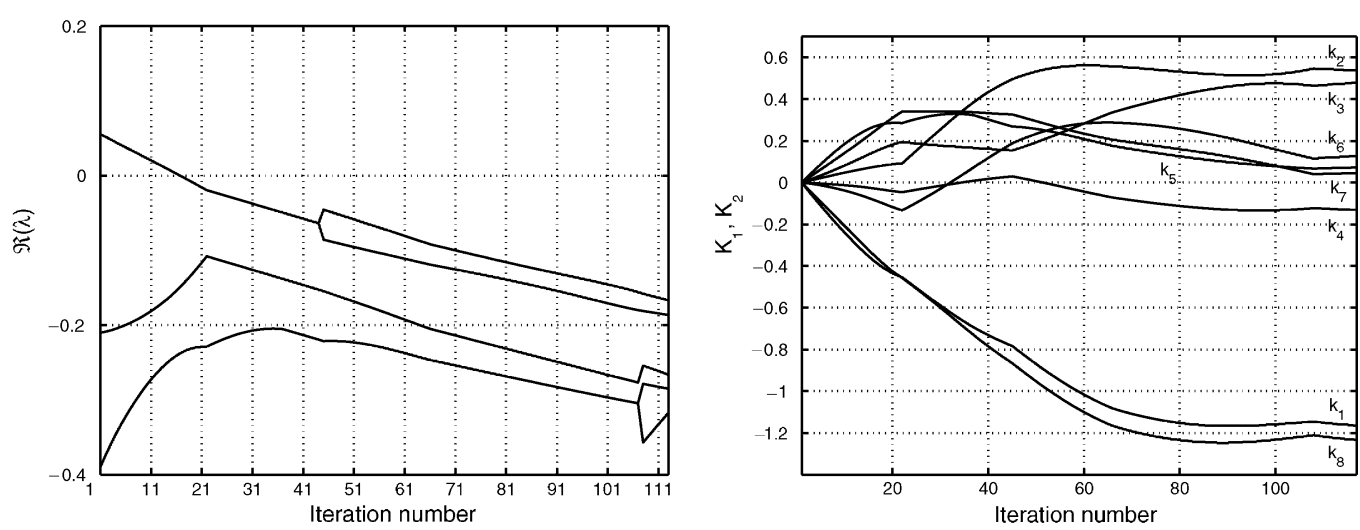

Fig. 9. Rightmost eigenvalues of (30) (left) and components of the feedback gains $K_{1}=\left[\begin{array}{llllll}k_{1} & k_{2} & k_{3} & k_{4}\end{array}\right]^{\mathrm{T}}$ and $K_{2}=\left[\begin{array}{llll}k_{5} & k_{6} & k_{7} & k_{8}\end{array}\right]^{\mathrm{T}}$ (right) as a function of the iterations of the continuous pole placement algorithm.

shown. Compared to system (28) a further reduction of the real parts of the dominant eigenvalues is possible, which is expected from the independent choice of both feedback gains. The algorithm also converges towards an optimum (w.r.t. all controller parameters), characterized by five coinciding real eigenvalues and hence, although there are eight controller parameters, it is not possible to further reduce sup $\Re(\lambda)$ by trying to control the real parts of more than four eigenvalues. This limitation is imposed by the structure of the controlled system, not by our algorithm. In this context we would like to emphasize first the danger of an 'over-parametrization' of the controller: having more degrees of freedom in the controller does not generally imply that more eigenvalues can be controlled or better stability results can be obtained. This is clearly illustrated in Michiels and Roose (2001), where a stabilization problem with four controller parameters was studied, which could analytically be reduced to three effective controller parameters. Second, although the continuous pole placement algorithm uses a local strategy in each iteration step and in the beginning only a few eigenvalues are controlled, generically all controller parameters are adapted in an iteration step when using formula (19), see, e.g. Fig. 9, and therefore the search space of the underlying optimization procedure (i.e. finding a minimum of $\sup \Re(\lambda)$ ) is generally not limited to a subspace of the parameter space where, e.g. some controller parameters are not used and remain zero.

As a second example we consider,

$\dot{x}=A_{1} x+A_{2} x\left(t-\tau_{1}\right)+\int_{t-\tau_{1}}^{t} A_{3} x(s) \mathrm{d} s+B u\left(t-\tau_{2}\right)$,

$u=K^{\mathrm{T}} x$,

where

$A_{1}=\left[\begin{array}{ccc}0.1 & 0 & 0 \\ 0.2 & 0 & -0.2 \\ 0.3 & 0.1 & -0.2\end{array}\right], \quad A_{2}=\left[\begin{array}{ccc}-0.2 & 0 & 0 \\ -0.4 & -0.2 & 0.4 \\ -0.4 & -0.1 & 0.2\end{array}\right]$,

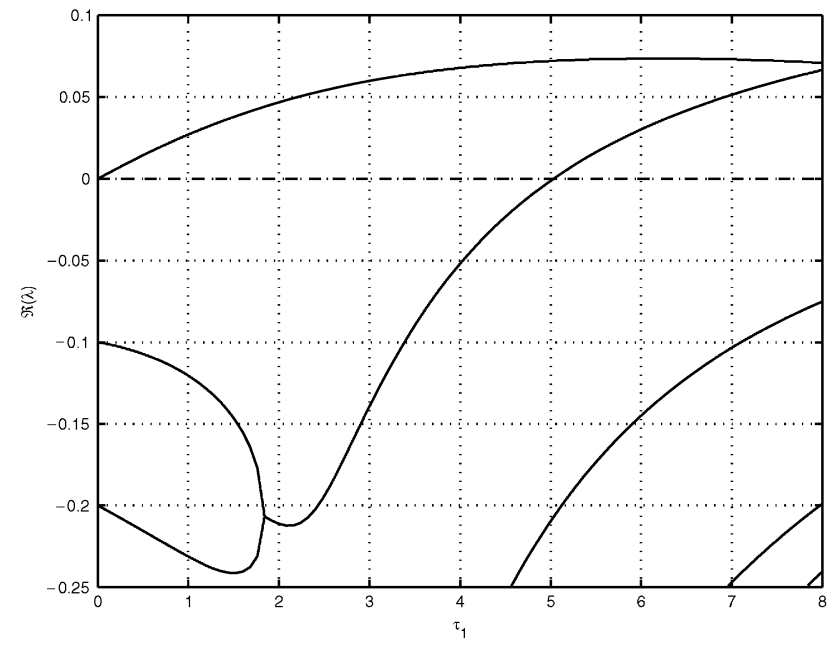

Fig. 10. Rightmost eigenvalues of the system (31)-(33) for $u=0$ as a function of the delay $\tau_{1}$. The solid lines correspond to the eigenvalues of (31). Eq. (33) has in addition a triple eigenvalue at zero (dashed line).

$$
\begin{aligned}
& A_{3}=\left[\begin{array}{ccc}
0.1 & -0.2 & 0 \\
0 & 0.1 & 0.1 \\
-0.1 & 0 & 0.1
\end{array}\right], \quad B=\left[\begin{array}{c}
0.1 \\
0 \\
0
\end{array}\right], \\
& \tau_{1}=6, \quad \tau_{2}=1 .
\end{aligned}
$$

We can deal with the distributed delay term because differentiation of (31) leads to the following equation with only discrete delays,

$$
\begin{aligned}
\dot{z}= & {\left[\begin{array}{cc}
0 & I \\
A_{3} & A_{1}
\end{array}\right] z+\left[\begin{array}{cc}
0 & 0 \\
-A_{3} & A_{2}
\end{array}\right] z\left(t-\tau_{1}\right) } \\
& +\left[\begin{array}{c}
0 \\
B K^{\mathrm{T}}
\end{array}\right] z\left(t-\tau_{2}\right),
\end{aligned}
$$



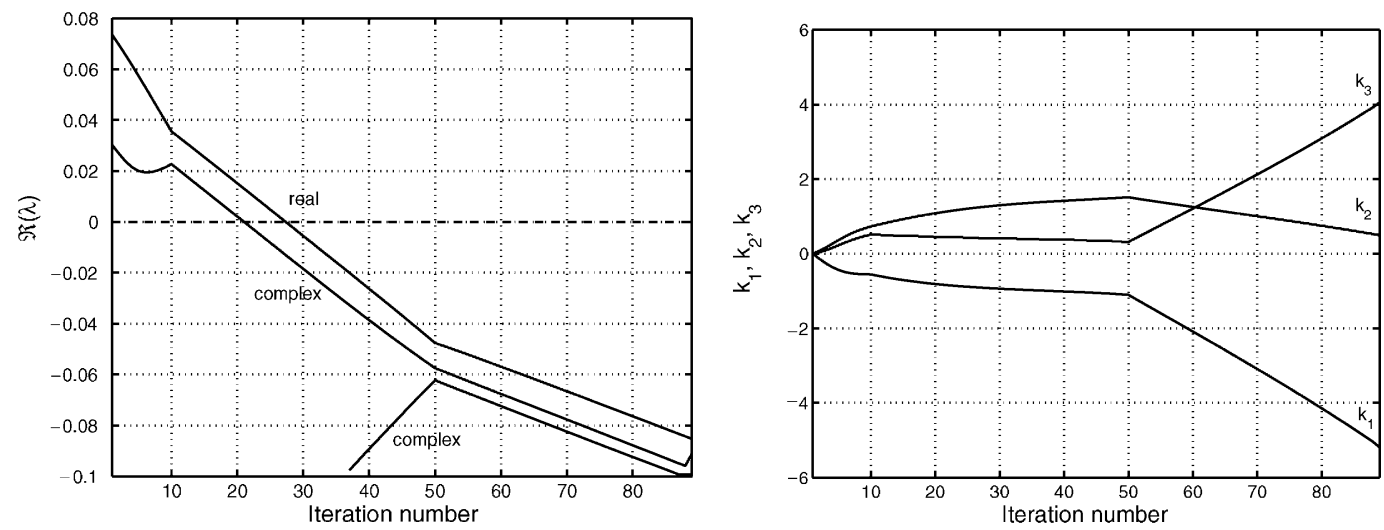

Fig. 11. Rightmost eigenvalues of (31)-(33) and components of the feedback gains $K=\left[\begin{array}{llll}k_{1} & k_{2} & k_{3}\end{array}\right]^{\mathrm{T}}$ (right) as a function of the iterations of the continuous pole placement algorithm.
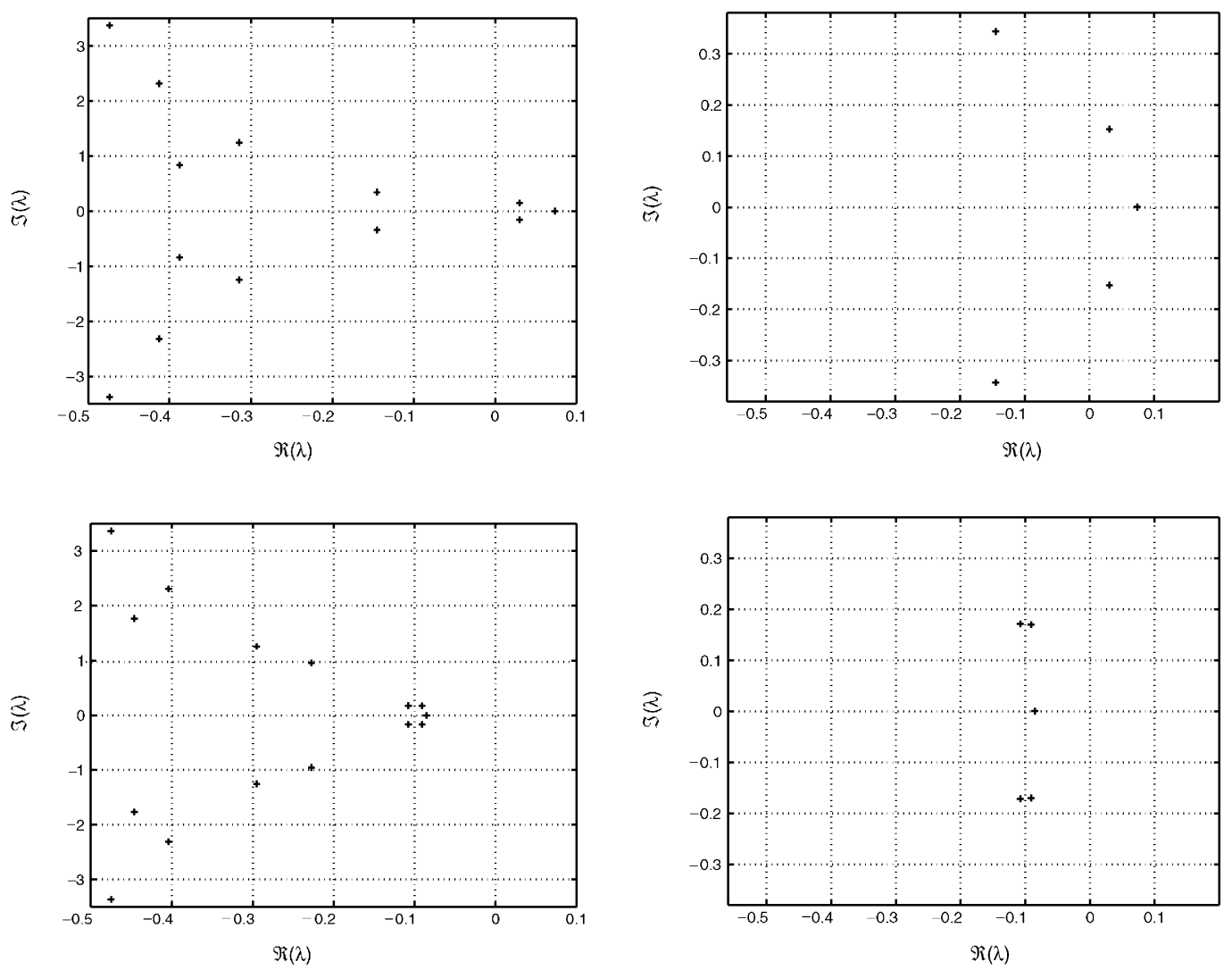

Fig. 12. Rightmost eigenvalues of (31) for $K=0$ (above) and for the final value of the feedback gain (Iteration $89, K=\left[\begin{array}{lll}-5.19 & 0.491 & 4.06\end{array}\right]^{\mathrm{T}}$ ) (below) on two different scales.

where $z=\left[\begin{array}{l}x \\ \dot{x}\end{array}\right]$. It is easy to show that the transformation from (31) to (33) introduces $n$ additional zero eigenvalues in the spectrum, with $n$ the dimension of the system. However, the continuous pole placement method can cope with this problem because the zero eigenvalues can simply be removed after applying step B of Algorithm 1 to (33). In Fig. 10, the eigenvalues of the uncontrolled system (31)-(33) are shown as a function of the delay $\tau_{1}$. For the nominal delay $\tau_{1}=6$, there are three eigen- values in the open right half plane. Iterations of the continuous pole placement algorithm are shown in Fig. 11. In Fig. 12, we depict the spectrum of (31) for $K=0$ and for the final value of the feedback gain. The continuous pole placement algorithm converges to an optimum characterized by five rightmost eigenvalues, a complex pair with multiplicity 2 and a real eigenvalue. Such a situation also occurred in the three-parameter problem discussed in Michiels and Roose (2001). 


\section{Concluding remarks}

In this paper, we have shown that the classical pole placement method for ODEs can be adapted to time-delay systems where the closed loop system is infinite dimensional and the number of degrees of freedom of the controller is finite. The method consists of controlling only the rightmost eigenvalues, which is possible because an algorithm to compute the rightmost eigenvalues of a linear time-delay system is available.

Further research includes the refinement of the continuous pole placement method, more specifically the development of strategies to determine the trajectories of the controlled eigenvalues in the complex plane, taking into account also robustness issues, and the study of the optima reached when the method breaks down. Also the comparison with methods based on finite spectrum assignment deserves further attention.

\section{Acknowledgements}

This research presents results of the Research Project OT 98/16 funded by the Research Council K.U. Leuven, of the Research Project G.0270.00 funded by the Fund for Scientific Research-Flanders (Belgium) and of the Research Project IUAP P4/02 funded by the programme on Interuniversity Poles of Attraction, initiated by the Belgian State, Prime Minister's Office for Science, Technology and Culture. The scientific responsibility rests with its authors. K. Engelborghs is a Postdoctoral Fellow of the Fund for Scientific Research-Flanders (Belgium).

\section{Appendix A. Continuity properties}

In the proof of Theorem 2, we use the following result, Lemma A-1 of Michiels et al. (1998):

Lemma 7. Let $f(\lambda)$ and the sequence $\left\{f_{n}(\lambda)\right\}_{n} \geqslant 1$ be analytic functions on an (open) domain $\mathscr{D} \subseteq \mathbb{C}$. Suppose that $\left\{f_{n}(\lambda)\right\}_{n \geqslant 1}$ converges uniformly to $f(\lambda)$ on the disc $D=\left\{\lambda:\left|\lambda-\lambda_{0}\right| \leqslant R\right\} \subset \mathscr{D}$ for some $R>0$ and that on this disc $\lambda_{0}$ is the only zero of $f(\lambda)$, with multiplicity $k \geqslant 0(k=0$ means no zeros in $D)$. Then there exists a number $N \in \mathbb{N}$ such that $\forall n \geqslant N, f_{n}(\lambda)$ has exactly $k$ zeros $\lambda_{n, 1}, \ldots, \lambda_{n, k}$ in $D$ and $\lim _{n \rightarrow \infty} \lambda_{n, j}=$ $\lambda_{0}, \forall j \in\{1, \ldots, k\}$.

With this lemma, continuity properties of the spectrum w.r.t. the feedback gain $K$ can easily be deduced:

Theorem 8. For the system $\dot{x}=A x+B K^{\mathrm{T}} x(t-\tau)$, the individual eigenvalues are continuous w.r.t. changes in the feedback gain K. Moreover $c_{K}=\sup \{\mathfrak{R}(\lambda)$ : $\operatorname{det}(\lambda I-$ $\left.\left.A-B K^{\mathrm{T}} \mathrm{e}^{-\lambda \tau}\right)=0\right\}$ is continuous w.r.t. $K$.

Proof. For an arbitrary eigenvalue $\lambda_{0}$ of

$\dot{x}=A x+B K^{\mathrm{T}} x(t-\tau)$

with multiplicity $k$, define for some (small) $R>0$ the set $D=\left\{\lambda \in \mathbb{C}:\left|\lambda-\lambda_{0}\right| \leqslant R\right\}$, containing no other eigenvalues. Further, consider an arbitrary sequence $\left\{K_{n}\right\}_{n \geqslant 1} \in \mathbb{R}^{1 \times n}$ with limit $K$. Since

$\operatorname{det}\left(\lambda I-A-B K_{n}^{\mathrm{T}} \mathrm{e}^{-\lambda \tau}\right) \rightarrow \operatorname{det}\left(\lambda I-A-B K^{\mathrm{T}} \mathrm{e}^{-\lambda \tau}\right)$

uniformly on $D$ as $n \rightarrow \infty$, we can apply Lemma 7 which states that the system

$\dot{x}=A x+B K_{n}^{\mathrm{T}} x(t-\tau)$

has exactly $k$ eigenvalues arbitrarily close to $\lambda_{0}$ as $n \rightarrow \infty$, which proves continuity of the individual eigenvalues.

The proof of the second statement is by contradiction. Since there exist eigenvalues of (A.1) with real part arbitrarily close to $c_{K}$, which are continuous w.r.t. changes in $K, \sup \Re(\lambda)$ cannot 'jump' to the left by applying small parameter changes, and therefore violation of the statement would imply: $\exists \varepsilon>0, \exists\left\{K_{n}\right\}_{n \geqslant 1} \rightarrow K, \exists\left\{\lambda_{n}\right\}_{n \geqslant 1}$, such that (A.2) has an eigenvalue $\lambda_{n}$ with $\Re\left(\lambda_{n}\right)>c_{K}+$ $\varepsilon$. From $\operatorname{det}\left(\lambda_{n} I-A-B K_{n}^{\mathrm{T}} \mathrm{e}^{-\lambda_{n} \tau}\right)=0$ it follows that $\left|\lambda_{n}\right| \leqslant\|A\|+\|B\| K_{\max } \mathrm{e}^{-c_{K} \tau}$ with $K_{\max }=\sup _{n \geqslant 1}\left\|K_{n}\right\|$. Define the set

$S=\left\{\lambda \in \mathbb{C}: \mathfrak{R}(\lambda) \geqslant c_{K}+\varepsilon,|\lambda| \leqslant\|A\|+\|B\| K_{\max } \mathrm{e}^{-c_{K} \tau}\right\}$.

When $S$ is empty, we have a contradiction. In the other case, clearly $\left\{\lambda_{n}\right\}_{n \geqslant 1} \in S$. Now define a closed disc $D$ containing $S$ and satisfying $\mathfrak{R}(\lambda) \geqslant c_{K}+\varepsilon / 2$ for $\lambda \in D$. Since for $n \rightarrow \infty$, the function $\operatorname{det}\left\{\lambda I-A-B K_{n}^{\mathrm{T}} \mathrm{e}^{-\lambda \tau}\right\}$ converges uniformly on $D$ to $\operatorname{det}\left(\lambda I-A-B K^{\mathrm{T}} \mathrm{e}^{-\lambda \tau}\right)$, which has no zeros in $D$, it follows from Lemma 7 that (A.2) cannot have eigenvalues in $S$ for large $n$ and we have again a contradiction.

\section{References}

Ackermann, J. (1972). Der entwurf linearer regelungssysteme im zustandsraum. Regelungstechnik und prozessdatenverarbeitung, 7 , 297-300.

Ben-Israel, A., \& Greville, T. N. E. (1974). Generalized inverses: theory and applications. Pure and applied mathematics. New York: Wiley.

Brethé, D., \& Loiseau, J. J. (1998). An effective algorithm for finite spectrum assignment of singe-input systems with delays. Mathematics and Computers in Simulation, 45(3-4), 339-348.

Cooke, K. L., \& Grossman, Z. (1982). Discrete delay, distributed delay and stability switches. Journal of Mathematical Analysis and Applications, 86, 592-627.

Dion, J. M., Dugard, L., \& Fliess, M. (Eds.). (1998). IFAC International Workshop on Linear Time Delay Systems, Grenoble, France. 
Dugard, L., \& Verriest, E. I. (1998). Stability and control of time-delay systems Lecture notes in control and information sciences, vol. 228. Berlin: Springer.

Engelborghs, K. (2000). DDE-BIFTOOL: a Matlab package for bifurcation analysis of delay differential equations. TW Report 305, Department of Computer Science, Katholieke Universiteit Leuven, Belgium, March. Available from http://www.cs.kuleuven.ac.be/ koen/delay/ddebiftool.shtml.

Engelborghs, K., Dambrine, M., \& Roose, D. (2001). Limitations of a class of stabilization methods for delay equations. IEEE Transactions on Automatic Control, 46(2), 336-339.

Engelborghs, K., \& Roose, D. (1999). Numerical computation of stability and detection of Hopf bifurcations of steady state solutions of delay differential equations. Advances in Computational Mathematics, 10(3-4), 271-289.

Hale, J. K., \& Verduyn Lunel, S. M. (1993). Introduction to functional differential equations Applied mathematical sciences, vol. 99. Berlin: Springer.

Hong-Jiong, T., \& Jiao-Xun, K. (1996). The numerical stability of linear multistep methods for delay differential equations with many delays. SIAM Journal on Numerical Analysis, 33(3), 883-889.

Hu, G. -D., Hu, G. -D., \& Liu, M. -Z. (1997). Estimation of numerically stable step-size for neutral delay-differential equations via spectral radius. Journal on Computational and Applied Mathematics, 78, 311-316.

in 't Hout, K. J. (1994). The stability of $\theta$-methods for systems of delay differential equations. Annals of Numerical Mathematics, 1, 323-334.

Kolmanovskii, V. B., \& Myshkis, A. (1999). Introduction to the theory and application of functional differential equations. Mathematics and its applications, vol. 463. Dordrecht: Kluwer Academic Publishers.

Kolmanovskii, V. B., \& Nosov, V. R. (1986). Stability of functional differential equations Mathematics in science and engineering, vol. 180. New York: Academic Press.

Manitius, A., \& Olbrot, A. (1979). Finite spectrum assignment problem for systems with delays. IEEE Transactions on Automatic Control, 24(4), 541-553.

Michiels, W., Engelborghs, K., Roose, D., \& Dochain, D. (1998). Sensitivity to infinitesimal delays in neutral equations. TW Report 286, Department of Computer Science, Katholieke Universiteit Leuven, Belgium, December; SIAM Journal on Control and Optimization, Accepted for publication.

Michiels, W., \& Roose, D. (2001). Limitations of delayed state feedback: a numerical study. TW Report 323, Department of Computer Science, Katholieke Universiteit Leuven, Belgium April; International Journal of Bifurcation and Chaos, Accepted for publication.

Olbrot, A. (1978). Stabilizability, detectability and spectrum assignment for linear autonomous systems with general time delays. IEEE Transactions on Automatic Control, 23(5), 605-618.

Palmor, Z. J. (1996). The control handbook. New York: CRC and IEEE Press, pp. 224-237.

Smith, O. J. (1957). Closer control of loops with dead time. Chemical Engineering Progress, 53, 217-219.

Van Assche, V., Dambrine, M., Lafay, J.-F., \& Richard, J.-P. (1999). Some problems arising in the implementation of distributed-delay control law. In Proceedings 38th IEEE CDC, paper FRM08-1.

Wang, Q. G., Lee, T. H., \& Tan, K. K. (1999). Finite spectrum assignment for time-delay systems. Lecture notes in control and information sciences, vol. 239. Berlin: Springer.

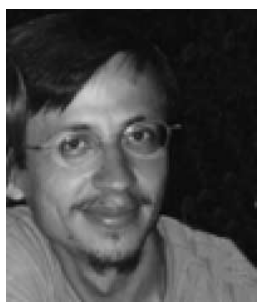

Wim V.A. Michiels was born in Mol, Belgium, on 9 January 1974. He obtained a M.S. degree in electrical engineering from the K.U. Leuven, Belgium, in 1997. Currently he is pursuing his $\mathrm{Ph}$.D. at the Department of Computer Science of the same university. His research interests include the stabilization and control of systems described by functional differential equations, nonlinear dynamical (control) systems and bifurcation analysis.

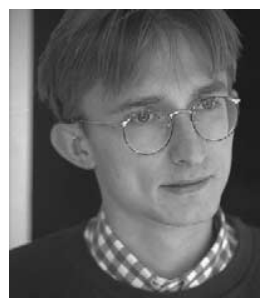

Koen Engelborghs was born on 2 January 1973. He obtained an engineering degree in Computer Science and Applied Mathematics in 1996 and a Ph.D. in Applied Sciences in 2000 both from the K.U. Leuven, Belgium. Currently he is a Postdoctoral Fellow of the Fund for Scientific ResearchFlanders (Belgium). His research interests include numerical algorithms and software for bifurcation analysis and control of partial differential equations and different types of delay differential equations. He is author of the software package DDE-BIFTOOL for numerical bifurcation analysis of delay differential equations.

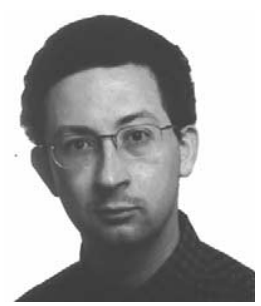

Patrick Vansevenant was born in Ostend, Belgium, on 19 September 1974. He obtained the degree of Complementary Studies in Applied Informatics from the K.U. Leuven, Belgium, in 1999. Currently he is researcher in the division Numerical Analysis and Applied Mathematics of the department of Computer Science of the same university. His research interests include the analysis and control of time-delay systems.

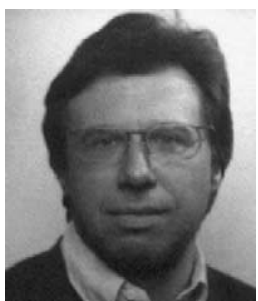

Dirk Roose was born on 25 December 1954. He obtained an engineering degree in computer science and applied mathematics in 1978 and his Ph.D. in Applied Sciences in 1985, both from the K.U. Leuven, Belgium. From 1989 till 1994 he has been assistant professor and since 1994 he is full professor at the Department of Computer Science of the same university, where he leads a research group on Scientific Computing. His research interests include nonlinear dynamical systems and bifurcation problems, and algorithms and software for scientific computing. He has co-authored over 100 publications on these subjects. He is editor of the book series 'Lecture Notes on Computational Science and Engineering', Springer Verlag and of the international journal 'Parallel Processing Letters'. 